\title{
Clinical spectrum and course of Dengue Fever during Pregnancy: Institutional experience from South India
}

\author{
${ }^{1}$ Rekha Gurumurthy, ${ }^{2}$ Gayathri KB, ${ }^{3}$ Seethamma R, ${ }^{4}$ Bhargav PRK
}

\begin{abstract}
:
Objective: Dengue infection is a major public health concern in tropical and subtropical World and an important differential diagnosis of fever during pregnancy influencing the obstetric outcome. In this context, we present our experience with clinical spectrum and course of Dengue fever in our series of obstetric cases.

Methods: A retrospective analysis of pregnant women diagnosed with dengue fever during pregnancy between August to October 2012 (15 months) was performed with regards to its clinical course, co-morbidities, obstetric outcome and maternal complications.

Results: The cohort included 19 cases of Dengue fever complicating pregnancy with one case of hemorrhagic

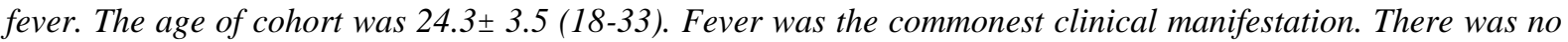
major bleeding diathesis. Preterm delivery and intrauterine death occurred in 3 cases each. All the complications and fetal deaths were related to hyperpyrexia. There was no maternal mortality. All the cases received only supportive therapy, antipyretics and ensuring adequate hydration. There was one neonatal death due to meconium aspiration syndrome.

Conclusions: 1) Early diagnosis of DF relies on high index of suspicion and supportive therapy with an aim to maintain normothermia is the cornerstone of therapy
\end{abstract}

(Key Words: Dengue fever; Pregnancy; HELLP Syndrome)

\section{Introduction:}

Dengue is a leading cause of death in tropics and sub tropics. With as many as 100 million people infected yearly (CDC), Dengue has emerged as a pandemic infection. Dengue fever (DF) is a viral disease spread by Aedes aegypti (Diptera : Culicidae) mosquito [1]. The unique feature of Aedes aegypti (AA) is thai it's a day time biting mosquito. Clinical spectrum of Dengue infection ranges from asymptomatic to Dengue hemorrhagic fever (DHF) with shock and death (WHO) [2]. The classical presentation of DF is initial febrile phase followed by critical phase, though the clinical course is highly unpredictable in an individual patient [3]. Diagnosis is clinical with laboratory evidence of flavivirus antigen, IgM antibody and treatment is primarily supportive. Prognosis becomes more adverse with co-existing infections such as malaria, tuberculosis etc., There are very few studies on DF in pregnancy from South India. In this context, we analysed and present our institutional experience on this issue.

Patient and Methods: This study was conducted in the Gynaecology and Obstetrics department of teritiary care teaching hospital in South India. The study design was retrospective analysis and duration of enrolled cases was August to October 2012 (15 months). The cohort included pregnant women diagnosed with dengue fever during pregnancy, who were analysed with regards to clinical course, co-morbidities, obstetric outcome and maternal complications. The clinical and laboratory details were collected from the database, hospital and labor room registry. This study complied with international ethical norms according to Helsinki Declaration - Ethical Principles for Medical Research Involving Human Subjects, 2004 and informed consent was sought from all the subjects [4]. Statistical analysis was done with SPSS software 12.0 version.

\section{Results:}

The cohort included 19 cases of DF complicating pregnancy, of whom 18 cases had DF and 1 case of DHF. The distribution of cases according to gestational period in first, second and third trimester were 1, 6 and 12 respectively. The age of cohort was $24.3 \pm 3.5(18-33)$. Ten cases were primigravidae and 9 were multigravidae. Fever was the commonest clinical manifestation in all the cases and symptoms were protean. Table 1 displays the entire range of major clinical features.

Inspite of thrombocytopenia, none of cases had major bleeding diathesis except from petechial hemorrhage in one case of DHF (Figure 1). Platelet count was less than $50,000 / \mathrm{mm}^{3} ; 50,000-100,000 / \mathrm{mm}^{3}$; $100,000-1,50,000 / \mathrm{mm}^{3}$ and more than $1,50,000 / \mathrm{mm}^{3}$ in $1,8,8$ and 2 cases respectively. Liver function test was within normal limits in most of the cases, but raised only 6 cases including in DHF. None developed jaundice. Seropositivity was as follows - Serum NS1 (non-specific antigen) was positive in 14 cases; IgM antibody in 2 cases and IgG antibody in 3 cases. Only the case with DHF with HELLP Syndrome had seropositivity of NS1 
antigen, $\operatorname{IgM}$ and $\operatorname{IgG}$ antibody. One case had co-existing malaria and one case was suffering from pulmonary tuberculosis, both of whom had prolonged illness with preterm labour.

The maternal outcome in our study was variable. The one DF case in first trimester had missed abortion. The obstetric outcome and gestational progression during $2^{\text {nd }}$ and $3^{\text {rd }}$ trimesters is depicted in Table 2 . The indications for cesarean section were non- reassuring fetal heart pattern on CTG and fetal distress. All the complications and fetal deaths were related to hyperpyrexia. There was no maternal mortality. All the cases received only supportive therapy, antipyretics and ensuring adequate hydration. All the cases were isolated during febrile phase and mosquito nets used to prevent cross infection.

Nine babies have borne alive. There was one neonatal death due to meconium aspiration syndrome. All the eight surviving neonates were asymptomatic. Four neonates were subjected to dengue antibody testing and were Ig G positive.

\section{Discussion:}

DF is caused by four dengue virus serotypes of the genus flavivirus and transmitted by Aedes aegypti mosquito. Infection provides immunity against the infecting viral serotype, but not against the other serotypes. The first attack of dengue fever (primary dengue) may predispose to much more severe illness, when infected with other serotypes (secondary dengue) [5]. Aedes Aegypti is a primary vector of dengue. Female Aedes Aegypti bites multiple people during each feeding period. The virus is transmitted to human through bite of infected female mosquito, which primarily infects the subjects with day time bite. Viremia is detected in 6-18 hours before onset of symptoms and disappears as the fever abates [6]. Infected humans act as the main carriers for 5 - 12 days after being infected.

The clinical manifestation of Dengue virus infection ranges from asymptomatic to symptomatic fever with DHF. According to WHO classification [7], it is classified in to 1) Asymptomatic; 2) Symptomatic - a) Undifferentiated fever, b) Dengue Fever - without hemorrhage and with hemorrhage and c) Dengue hemorrhagic fever - without shock and Shock syndrome (DSS). In our study, 18 cases had DF without hemorrhage i.e., $2 b$ category and only one DHF case without shock ( $2 c$ category). DF is an acute febrile illness lasting for 2-7 days (sometimes with two peaks) with two or more of the following clinical manifestations: headache, retro -orbital pain, myalgia/arthralgia, rash, hemorrhagic manifestation, leukopenia. In a review by Sirinavin et al.[8] 13/14 (93\%) cases had a typical presentation of abrupt fever accompanied by headache, retroorbital pain, muscle aches and thrombocytopenia. Phuphong et al [9] reported a similar typical course in a case report. Similarly as seen in this study, $100 \%$ of cases had fever and other constitutional symptoms in most of the cases. DHF is usually manifested by petechiae, ecchymosis or purpura; bleeding from mucosa (mostly epistaxis or bleeding from gums or injection sites), thrombocytopaenia (platelets $100,000 / \mathrm{mm}^{3}$ or less) and positive tourniquet test. Evidence of plasma leakage due to increased capillary permeability is manifested by one or more of the following: More than 20\% rise in haemotocrit; More than 20\% drop in haemotocrit following treatment as compared to baseline; signs of plasma leakage (pleural effusion, ascites or hypoproteinaemia). In one case of DHF, minimum platelet count was $45000 / \mathrm{mm}^{3}$ and suffered from petechial rash diagnosed by positive tourniquet test. Dengue shock syndrome (DSS) is characterized by DHF and signs of circulatory failure.

The clinical course of DF/ DHF is highly unpredictable. Most patients have a febrile phase lasting $2-7$ days, followed by a critical phase of 2-3 days duration. During this phase, the patient is afebrile, and is at risk of developing DHF/DSS, which may prove fatal if prompt and appropriate treatment is not instituted. Rash could be diffuse, vesicular or maculopapular. The diagnosis is based on clinical suspicion and laboratory confirmation of seropositivity.. Commercially available kits isolate a nonspecific antigen of the flavivirus group (NS 1 antigen), IgM, IgG. The most frequently used serologic tests are the hemagglutination inhibition (HI) assay and IgG or IgM enzyme immunoassays. The IgM immunoassay (MAC-ELISA or equivalent) is the most commonly used for rapid confirmation of the diagnosis [10]. NS1 antigen is positive in the initial 2-7 days of fever with a specificity of $90 \%$. In the present study, NS1 was positive in $14 / 19(74 \%)$ of cases and management of a confirmed case of Dengue is largely symptomatic with attention to hydration and temperature control with tepid sponging and antipyretics.

Increasing age and parity confers immunity against the serotypes of dengue in endemic areas. A study conducted by Perret et al. in parturients of highly endemic area concluded that higher seropositivity rate and preexisting protective immunity with advancing maternal age [6]. Carles et al, [11] in their review of 38 cases, indicate a significant increase in prematurity and fetal death in DF. Ismail et al, also reported noted a 50\% prematurity rate and reported three maternal deaths out of 16 cases [12]. Most of the cases of prematurity, intrauterine deaths and miscarriages were related to hyperpyrexia. Perrett et al. concluded in a study that serious DF occurs in newborn only when the mother is at or near term with insufficient time for the maternal production of protective antibodies [6]. Isolation of patients and use of mosquito nets is very essential to prevent crossinfection. Transplacental spread of disease and placental damage due to dengue is not well studied issue. 


\section{Conclusions:}

1) Early diagnosis of DF relies on high index of suspicion as nonspecific clinical features, usual physiologic changes in pregnancy and broad differential diagnosis precludes it.

2) Supportive therapy with an aim to maintain normothermia is the cornerstone of therapy.

3) In absence of other complications, DF perse does not appear to be an indication for obstetrical intervention.

4) Pregnant women should be counseled about preventive strategies against contracting DF

\section{References:}

[1] Chitra TV, Panicker JS. Vector Borne Dis 2011;48: 210-213

[2] Guidelines for treatment of Dengue fever/Dengue haemorrhagic fever in small hospitals: World Health Organization, Regional Office for South-East Asia, New Delhi;2000

[3] Gubler DJ. Dengue and dengue hemorrhagic fever. Clin Microbiol Rev 1998;11:480

[4] World Medical Association. Declaration of Helsinki. Note of Clarification on Paragraph 30 added by the WMA General Assembly. Tokyo, 2004

[5] Vaughn DW, Green S, Kalayanarooj S, Innis BL, Nimmannitya S, Suntayakorn S et al. Dengue in the early febrile phase: viremia and antibody responses. J Infect Dis 1997;176:322-330

[6] Perret C, Chanthavanich P, Pengsaa K, Limkittikul $K$, Hutajaroen P, Bunn JE et al. Dengue infection during pregnancy and transplacental antibody transfer in Thai mothers. J Infect 2005;51:287-93

[7] Deen JL, Harris E, Wills B, Balmaseda A, Hammond SN, Rocha C, et al. The WHO dengue classification and case definitions: time for a reassessment. Lancet $2006 ; 368: 170-3$

[8] Sirinavin S, Nuntnarumit P, Supapannachart S, Boonkasidecha S, Techasaensiri C, Yoksarn S. Vertical dengue infection: case reports and review. Pediatr Infect Dis J 2004;23:1042-7

[9] Phupong V. Dengue fever in pregnancy: a case report. BMC Pregnancy Childbirth 2001;1:7

[10] Blacksell SD, Doust JA, Newton PN, Peacock SJ, Day NPJ, Dondorp AN. A systematic review and meta-analysis of the diagnostic accuracy of rapid immunochromatographic assays for the detection of dengue virus IgM antibodies during acute infection. Trans $\mathrm{R}$ Soc Trop Med Hygiene 2006;100:775-84

[11] Carles G, Talarmin A, Peneau C, Bertsch M. Dengue fever and pregnancy. A study of 38 cases in French Guiana. J Gynecol Obstet Biol Reprod (Paris) 2000;29:758-62

[12] Ismail NAM, Kampan M, Mahdy ZA, Jamil MA, Razi ZRM. Dengue in Pregnancy. Southeast Asia J Trop Med 2006; 37:681-3

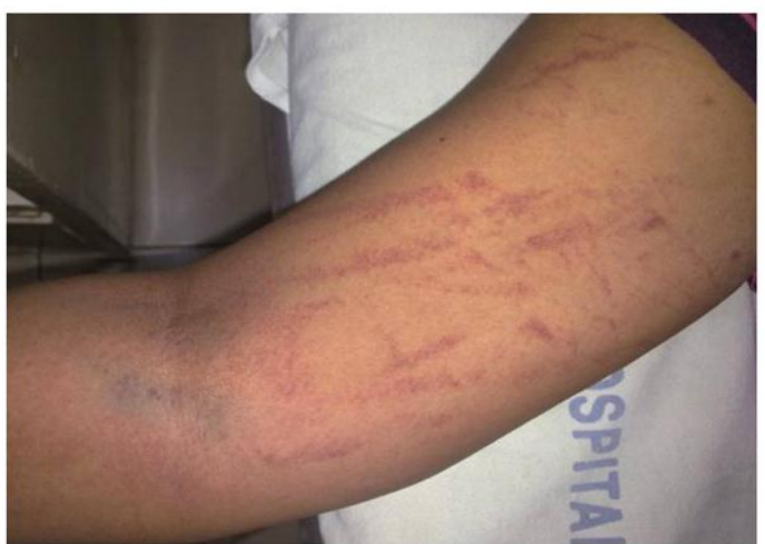

Figure 1: Clinical photograph showing petechial rash over arm on tourniquet test

Table 1: The spectrum of clinical features amongst DF patients

\begin{tabular}{|l|l|}
\hline Fever & 19 \\
\hline Myalgia & 15 \\
\hline Headache & 10 \\
\hline Periorbital pain & 9 \\
\hline Maculopapular rash & 4 \\
\hline Petechial haemorrhage & 1 \\
\hline Hemiparesis with disorientation & 1 \\
\hline
\end{tabular}

Table 2: Obstetric outcome and gestational progression during $2^{\text {nd }}$ and $3^{\text {rd }}$ trimesters

\begin{tabular}{|l|l|l|l|}
\hline $\mathbf{2}^{\text {nd }}$ Trimester & $\mathbf{N = 6}$ & $\mathbf{3}^{\text {rd }}$ Trimester & $\mathbf{N}=\mathbf{1 2}$ \\
\hline No. of cases reaching term & 3 & Intra uterine death & 1 \\
\hline Intra-uterine death & 2 & Pre-term vaginal live births & 3 \\
\hline Induced abortion for FA * & 1 & Term vaginal live births & 4 \\
\hline- & - & LSCS $*$ & 4 \\
\hline
\end{tabular}

$\mathrm{FA}=$ Fetal anamolies; LSCS $=$ Lower segment Caesarian section 